\title{
Avaliação nutricional e envelhecimento
}

\author{
Nutritional evaluation and ageing
}

Lílian Ramos SAMPAIO'

\section{R E S U M O}

A avaliação do estado nutricional do idoso é considerada complexa em razão da influência de uma série de fatores, os quais necessitam ser investigados, detalhadamente, visando diagnóstico nutricional acurado, que possibilite intervenção nutricional adequada. Alterações fisiológicas, processos patológicos crônicos e situações individuais que ocorrem com o envelhecimento, geralmente interferem no estado nutricional do indivíduo. Portanto impõe-se uma associação de indicadores nutricionais e a utilização de padrões de referência os mais específicos possíveis para realizar-se tal avaliação. Com o objetivo de contribuir ao estudo nutricional do indivíduo idoso, este trabalho discute os principais aspectos relacionados à avaliação do estado nutricional, com ênfase nos indicadores e critérios de avaliação mais apropriados para esse grupo etário, considerando-se as modificações inerentes ao processo de envelhecimento.

Termos de indexação: avaliação nutricional, antropometria, idoso, nutrição, envelhecimento da população.

\section{A B S T R A C T}

The evaluation of the nutritional status of the elderly is complex due to the influence of several factors, which require detailed investigation, in order to reach an exact and accurate diagnosis that will enable the prescription of adequate nutrition. There are physiological alterations, chronic pathological processes and individual situations that occur with ageing, which generally interfere with the individual's nutritional status. This requires the association of nutritional indicators and the use of the most specific reference patterns, when performing such evaluation. To contribute to the nutritional study of the elderly, this paper discusses the main aspects related to the evaluation of their nutritional status, emphasizing the most appropriate evaluation criteria and indicators for such age group, and considering the changes inherent in the ageing process.

Index terms: nutritional evaluation, anthropometry, aged, nutrition, demographic aging.

\footnotetext{
1 Departamento de Ciências da Nutrição, Escola de Nutrição, Universidade Federal da Bahia. Av. Araújo Pinho, 32, Canela, 40110-150, Salvador, BA, Brasil.E-mail: lramos@ufba.br
} 


\section{N T R O D U Ç Ã O}

Tem-se registrado envelhecimento mundial das populações ${ }^{1}$. Este fato gera maior necessidade em aprofundar a compreensão sobre o papel da nutrição na promoção e manutenção da independência e autonomia dos idosos.

As principais funções que o profissional da nutrição desempenha nesse contexto são: a identificação de indivíduos em risco para desenvolver doenças crônicas não-transmissíveis e a intervenção alimentar para a prevenção e controle de enfermidades ${ }^{2}$.

A incidência de doenças crônicas é alta nos indivíduos idosos e o risco de desenvolvê-las ou de torná-las mais graves, levando a incapacidades, deve ser identificado precocemente 3 . Isso é possível a partir da avaliação adequada do estado nutricional, a qual deve considerar as especificidades de cada indivíduo idoso, uma vez que este é parte de um grupo bastante heterogêneo ${ }^{4}$.

A determinação do diagnóstico nutricional e a identificação dos fatores que contribuem para tal diagnóstico no indivíduo idoso são, portanto, processos fundamentais, mas complexos. A complexidade se deve à ocorrência de diversas alterações, tanto fisiológicas quanto patológicas, além de modificações de aspectos econômicos e de estilo de vida, entre outros, com o avançar da idade 2,5 .

Portanto, com o objetivo de contribuir para o estudo nutricional do indivíduo idoso, este trabalho discute os principais aspectos relacionados à avaliação do seu estado nutricional, dando ênfase aos indicadores e critérios de avaliação mais apropriados para esse grupo etário, considerando as modificações inerentes ao processo de envelhecimento.

\section{INDICADORES DE DIAGNÓSTICO NUTRICIONAL}

\section{Exames bioquímicos}

Os exames bioquímicos mais comumente utilizados para a avaliação nutricional do idoso são: albumina, transferrina, hematócrito, hemoglobina, contagem total de linfócitos, colesterol total e frações ${ }^{2,6}$. Atenção é essencial na interpretação desses exames, pois, apesar de detectar problemas nutricionais precocemente, os indicadores bioquímicos podem ter sido influenciados por enfermidades, uso de drogas ou estresse, condições estas bastante freqüentes no indivíduo idoso ${ }^{2,7}$.

\section{Sinais clínicos nutricionais}

Os sinais clínicos apresentam baixa especificidade para identificação de problemas nutricionais no indivíduo idoso. Isto ocorre porque muitos sinais podem ter como causa alterações fisiológicas decorrentes do processo de envelhecimento ou, ainda, a instalação de algum processo patológico não-nutricional. A púrpura senil, que geralmente é conseqüência do envelhecimento da pele pode, por exemplo, ser confundida com hipovitaminose. Um outro achado bastante comum é a cegueira noturna, que pode ter como causa a presença de catarata e não a deficiência de vitamina $A^{8}$.

A investigação dos sinais de carência ou excesso, assim como dos indicadores de deficiência nutricional específica, deve fazer parte da avaliação do estado nutricional do idoso; entretanto, este exame necessita ser interpretado a partir da história clínica e em associação com indicadores bioquímicos, antropometria e/ou qualquer outro método apropriado 8,7 .

\section{Antropometria}

O indicador antropométrico é essencial na avaliação nutricional geriátrica; entretanto algumas alterações que ocorrem com o envelhecimento podem comprometer a determinação de um diagnóstico antropométrico acurado e preciso, caso cuidados específicos não sejam tomados no sentido de neutralizar ou amenizar o efeito dessas alterações sobre a avaliação $0^{5,9}$. Serão discutidas a 
seguir as principais alterações que podem interferir na avaliação nutricional antropométrica.

Em relação a altura, estudos apontam uma redução na altura com a idade. Perissinotto et al. ${ }^{5}$ encontraram um decréscimo de $2 \mathrm{~cm} /$ década a $3 \mathrm{~cm} /$ década, enquanto que o Euronut Seneca Study quantificou uma diminuição na altura de $1 \mathrm{~cm}$ a $2 \mathrm{~cm}$ em 4 anos. Este declínio se inicia por volta dos 40 anos e torna-se mais acentuado com o avançar da idade 5 . As razões para este declínio são: achatamento das vértebras, redução dos discos intervertebrais, cifose dorsal, escoliose, arqueamento dos membros inferiores e/ou achatamento do arco plantar.

Sendo assim, recomenda-se a estimativa da altura de indivíduos com idade igual ou superior a 60 anos, tendo em vista que, por alguma(s) dessas alterações, a altura já sofreu algum declínio ${ }^{10}$.

A altura pode ser estimada a partir do comprimento da perna (altura do joelho ou knee height), envergadura do braço ou da altura recumbente. No entanto, em virtude da rigidez nas articulações, torna-se difícil o idoso se posicionar adequadamente para a identificação das duas últimas medidas. Portanto, o comprimento da perna tem sido recomendado para estimar a altura de idosos a partir das equações a seguir, pois esta medida apresenta as seguintes vantagens: possibilita estimar a altura de pacientes acamados e deitados, quando utilizadas as equações de Chumlea et al. ${ }^{10}$; muda pouco com a idade; apresenta alta correlação com estatura; possibilita estimar o peso corporal, quando associada a outras medidas (detalhes na discussão sobre o peso) e utilizadas as equações de Chumlea et al. ${ }^{11}$.

Esta medida deve ser verificada com o auxílio de uma régua antropométrica ou de um infantômetro com haste de metal. O indivíduo deve ficar com a perna dobrada, formando um ângulo de $90^{\circ} \mathrm{com}$ o joelho. A parte fixa da régua é colocada embaixo do calcanhar e a móvel é trazida para dois a três dedos da patela. Pode ser verificada com o indivíduo sentado ou deitado e a leitura é feita no milímetro mais próximo ${ }^{10}$.

Equações para estimar estatura de idosos a partir do comprimento da perna ${ }^{10}$.

Homem: [2,02 x comprimento da perna] $-[0,04 \times$ idade $]+64,19$

Mulher: [1,83 x comprimento da perna] $-[0,24 \times$ idade $]+84,88$

Para o indicador peso, estudos mostram que o homem ganha peso até os 65 anos de idade e, a partir daí, passa a perder, enquanto que a mulher aumenta de peso até os 75 anos e, apenas a partir desta idade, ela começa com a perda ponderal ${ }^{12,13}$. As causas principais são a perda de água corporal e a redução no peso das vísceras, além da redução de tecido muscular.

Na situação em que o indivíduo estiver impossibilitado de ser pesado com auxílio de balança, é possível estimar o peso corporal. Existem equações para este propósito, entretanto elas apresentam importantes limitações como a elevada margem de erro e a necessidade de várias medidas corporais, ou seja: circunferências da panturrilha $(C P)$ e do braço $(C B)$, o comprimento da perna e a prega cutânea subescapular (PCSE). Enquanto equações mais simplificadas e acuradas não forem elaboradas, recomenda-se as propostas por Chumlea ${ }^{11}$, possibilitando a estimativa desta medida de suma importância na avaliação do estado nutricional.

Equações para estimativa do peso a partir de medidas corporais ${ }^{11}$.

Homem: $(0,98 \times C P)+(1.16 \times$ comprimento da perna $)+(1,73 \times \mathrm{CB})+(0,37 \times \mathrm{PCSE})-$ 81,69

Mulher: $(1,27 \times C P)+(0,87 \times$ comprimento da perna $)+(0,98 \times C B)+(0,4 \times P C S E)-$ 62,35

\section{Composição corporal}

Com o envelhecimento, ocorre aumento na gordura corporal total e redução do tecido 
muscular. Essas modificações no tecido muscular ocorrem, principalmente, em virtude da diminuição da atividade física e da taxa metabólica basal ${ }^{5,14}$.

A reserva de tecido muscular pode ser estimada antropometricamente pela circunferência muscular do braço (CMB) e circunferência da panturrilha. $A C M B$, apesar de não levar em consideração a irregularidade no formato dos tecidos do braço, deve ser o indicador de escolha, tendo em vista que o Third National Health and Nutrition Examination Survey (NHANES III) ${ }^{15}$ apresenta dados de referência para este indicador (veja discussão no item sobre dados de referência). A circunferência da panturrilha é considerada um indicador sensível de alterações musculares no indivíduo idoso e deve ser utilizada para monitoração dessas alterações ${ }^{16}$. Quanto ao tecido adiposo, apesar das limitações, recomenda-se a utilização da prega cutânea triciptal $(P C T)^{15}$. Equação para estimar tecido muscular ${ }^{17}$ : $\mathrm{CMB}=\mathrm{CB}-(\pi \times \mathrm{PCT})$

\section{Distribuição de gordura corporal}

Com o envelhecimento, além do aumento da gordura corporal, observa-se redistribuição desse tecido, havendo diminuição nos membros e acúmulo preferencialmente na região abdominal5,18.

A identificação do tipo de distribuição de gordura corporal é de suma importância pois o acúmulo de gordura na região abdominal apresenta estreita relação com alterações metabólicas, as quais podem desencadear o aparecimento de enfermidades como as cardiovasculares e diabetes mellitus ${ }^{19,20}$. Estudos evidenciam que, com o avançar da idade, ocorre aumento da gordura visceral e que a relação entre acúmulo de gordura abdominal e alterações metabólicas se mantém com a idade ${ }^{21}$.

Portanto, apesar de não diagnosticar estado nutricional, a utilização de medidas antropométricas para identificação do tipo de distribuição da gordura corporal, é fundamental na avaliação de risco para doenças associadas à obesidade.
A medida da circunferência da cintura tem sido proposta como um dos melhores preditores antropométricos de gordura visceral19,22,23. Essa medida deve ser verificada entre as costelas inferiores e as cristas ilíacas. A leitura é feita no momento da expiração.

A medida da circunferência da cintura isoladamente é analisada a partir dos pontos de corte sugeridos pela Organização Mundial da Saúde (OMS)24 (Tabela 1).

Tabela 1. Valores da CC(cm) considerados como risco para doenças associadas à obesidade.

\begin{tabular}{lcc}
\hline & Risco elevado & Risco muito elevado \\
\hline Mulheres & $\geq 80$ & $\geq 88$ \\
Homens & $\geq 94$ & $\geq 102$ \\
\hline
\end{tabular}

Fonte: World Health Organization ${ }^{24}$

Outro indicador bastante utilizado na avaliação do tipo de distribuição de gordura corporal é a razão cintura-quadril (RCQ); entretanto, estudos têm encontrado associação moderada entre este indicador e o acúmulo abdominal de gordura ${ }^{19,25}$. A medida do quadril deve ser feita no nível da sínfise púbica, com a fita métrica circundando o quadril na parte mais saliente entre a cintura e a coxa, com o indivíduo usando roupas finas. Realiza-se a leitura no milímetro mais próximo. Os pontos de corte mais utilizados para avaliação de risco, segundo a RCQ (Tabela 2).

Tabela 2. Valores de risco da RCQ segundo o sexo.

\begin{tabular}{llc}
\hline & Ginóide & Andróide \\
\hline Masculino & $<1,00$ & $>1,00$ \\
Feminino & $<0,80$ & $>0,80$ \\
\hline
\end{tabular}

Fonte: Bray ${ }^{26}$

Uma das principais limitações desses dois indicadores de distribuição de gordura corporal é a ausência de pontos de corte específicos para a 
população idosa. Utilizam-se, até o momento, as recomendações propostas para os adultos jovens, sem considerar as alterações na distribuição de gordura inerentes ao processo de envelhecimento.

Por fim, existem alguns estudos avaliando a predição de gordura visceral a partir da utilização da medida do diâmetro abdominal ${ }^{19,27}$. Essas investigações iniciais apontam esta medida como o melhor preditor de tecido visceral; porém, além dos estudos serem escassos, ainda não foi preconizado o ponto de corte considerado como risco para o desenvolvimento de enfermidades. A medida do diâmetro abdominal é realizada com o auxílio de um antropômetro portátil (Sliding-beam - Holtain, Ltd., Dyfed. Wales, UK). É verificado com o indivíduo deitado, os braços relaxados ao longo do corpo e as pernas estendidas. A haste fixa do antropômetro é colocada embaixo das costas do indivíduo e a móvel é trazida até a marca abdominal feita entre as cristas ilíacas, no nível do umbigo. A leitura é feita no milímetro mais próximo, no final da expiração.

\section{Massa corporal}

O Índice de Massa Corporal, que é calculado a partir do peso (em $\mathrm{kg}$ ) dividido pelo quadrado da altura em metros (Peso/Altura ${ }^{2}-\mathrm{kg} / \mathrm{m}^{2}$ ), tem como finalidade avaliar a massa corporal em relação à altura. Apesar do seu uso freqüente, ele deve estar sempre associado a outros indicadores, tendo em vista que o mesmo não reflete a distribuição regional de gordura ou qualquer mudança na distribuição de gordura ocorrida com o processo de envelhecimento, sendo considerado, portanto, um indicador pobre para avaliar riscos em idosos ${ }^{5}$.

Não há consenso sobre a definição do que seja um IMC elevado ou baixo para o indivíduo idoso. Apesar da OMS ${ }^{24}$ sugerir para avaliação do idoso a utilização dos pontos de corte propostos para o adulto jovem $\left(\mathrm{IMC}<18,5 \mathrm{~kg} / \mathrm{m}^{2}=\right.$ magreza; $I M C \geq 30 \mathrm{~kg} / \mathrm{m}^{2}=$ obesidade), supõe-se que valores superiores sejam mais adequados, já que o idoso necessita de uma reserva maior no sentido de prevenir a desnutrição. Em pesquisas populacionais, a maioria dos estudos utiliza o IMC médio interno de suas amostras. Na prática clínica geriátrica, o $\mathrm{IMC}<20 \mathrm{~kg} / \mathrm{m}^{2}$ é o ponto de corte adotado para classificar o idoso como apresentando magreza ${ }^{5}$. Este critério é utilizado para que indivíduos em risco nutricional sejam detectados precocemente. Para o diagnóstico da obesidade, utiliza-se o ponto de corte sugerido pela $\mathrm{OMS}^{24}$, associando o IMC à prega cutânea triciptal.

\section{Alterações nos tecidos de elasticidade e compressibilidade}

Com a perda de água corporal, redução do tecido muscular e diminuição da gordura nos membros, ocorre perda da elasticidade e maior compressibilidade dos tecidos 9 . Isto interfere principalmente na verificação das pregas cutâneas, levando a uma dificuldade maior na separação do tecido muscular do adiposo, assim como na medida das circunferências, especialmente no ajustamento da fita métrica e na identificação do local correto para realizar a medida. Portanto, em razão da importância destas medidas na estimativa do tecido adiposo e de sua distribuição corporal, o seu uso é recomendado desde que se leve em consideração as limitações citadas anteriormente.

\section{Dados de referência não muito específicos para população idosa}

A avaliação nutricional do idoso apresenta limitações também em relação aos dados e padrões de referência para as medidas antropométricas. Nos países em desenvolvimento, inexistem dados ou padrões para esse grupo etário. Nos países desenvolvidos, os estudos para a construção desses dados e padrões foram realizados a partir de amostras representativas, porém, com limite superior de idade ${ }^{17,28,29}$. 
Atualmente, a OMS recomenda para a avaliação nutricional do indivíduo idoso, inclusive nas nações em desenvolvimento, a utilização dos dados de referência do NHANES III (Tabelas 3 e 4), tendo em vista que este estudo foi construído a partir de uma amostra de 5700 idosos norte-americanos não institucionalizados, sem limite superior de idade (60 e mais) e com metodologia padronizada ${ }^{15}$. As Tabelas 1 e 2 apresentam dados para avaliação da PCT, CB e CMB.

\section{INDICADORES QUE EXPLICAM O DIAGNÓSTICONUTRICIONAL}

É fundamental que, além da identificação do diagnóstico nutricional, sejam investigados os fatores que podem explicar o diagnóstico encontrado. Isto permitirá uma intervenção nutricional adequada. Para esse grupo etário, os aspectos que mais interferem no estado nutricional estão apresentados no Quadro 1.

Tabela 3. Circunferência do braço, prega cutânea tricipital e circunferência muscular do braço para homens de 60 anos ou mais, avaliados no Third National Health and Nutrition Examination Survey - NHANES III (1988-1994).

\begin{tabular}{|c|c|c|c|c|c|c|c|c|c|}
\hline \multirow{2}{*}{$\begin{array}{l}\text { Variáveis e grupo de } \\
\text { idade }^{a}\end{array}$} & \multirow{2}{*}{$n$} & \multirow{2}{*}{ Média $\pm E P^{b}$} & \multicolumn{7}{|c|}{ Percentil } \\
\hline & & & 10 & 15 & 25 & 50 & 75 & 85 & 90 \\
\hline \multicolumn{10}{|l|}{$\mathrm{CB}(\mathrm{cm})$} \\
\hline $60-69$ & 1126 & $32,8 \pm 0,15$ & 28,4 & 29,2 & 30,6 & 32,7 & 35,2 & 36,2 & 37,0 \\
\hline $70-79$ & 832 & $31,5 \pm 0,17$ & 27,5 & 28,2 & 29,3 & 31,3 & 33,4 & 35,1 & 36,1 \\
\hline$\geq 80$ & 642 & $29,5 \pm 0,19$ & 25,5 & 26,2 & 27,3 & 29,5 & 31,5 & 32,6 & 33,3 \\
\hline \multicolumn{10}{|l|}{ PCT (mm) } \\
\hline $60-69$ & 1122 & $14,2 \pm 0,25$ & 7,7 & 8,5 & 10,1 & 12,7 & 17,1 & 20,2 & 23,1 \\
\hline $70-79$ & 825 & $13,4 \pm 0,28$ & 7,3 & 7,8 & 9,0 & 12,4 & 16,0 & 18,8 & 20,6 \\
\hline$\geq 80$ & 641 & $12,0 \pm 0,28$ & 6,6 & 7,6 & 8,7 & 11,2 & 13,8 & 16,2 & 18,0 \\
\hline \multicolumn{10}{|l|}{ CMB (cm) } \\
\hline $60-69$ & 1119 & $28,3 \pm 0,13$ & 24,9 & 25,6 & 26,7 & 28,4 & 30,0 & 30,9 & 31,4 \\
\hline $70-79$ & 824 & $27,3 \pm 0,14$ & 24,4 & 24,8 & 25,6 & 27,2 & 28,9 & 30,0 & 30,5 \\
\hline$\geq 80$ & 639 & $25,7 \pm 0,16$ & 22,6 & 23,2 & 24,0 & 25,7 & 27,5 & 28,2 & 28,8 \\
\hline
\end{tabular}

(a) Todos os grupos étnicos; (b) Erro-padrão. $\mathrm{CB}=$ circunferência do braço; $\mathrm{PCT}=$ prega cutânea tricipital; $\mathrm{CMB}=$ circunferência muscular do braço. Fonte: Kuczmarski et al. ${ }^{15}$.

Tabela 4. Circunferência do braço, prega cutânea tricipital e circunferência muscular do braço para mulheres de 60 anos ou mais, avaliadas no Third National Health and Nutrition Examination Survey - NHANES III (1988-1994).

\begin{tabular}{|c|c|c|c|c|c|c|c|c|c|}
\hline \multirow{2}{*}{$\begin{array}{l}\text { Variáveis e grupo de } \\
\text { idade }^{a}\end{array}$} & \multirow{2}{*}{$n$} & \multirow{2}{*}{ Média $\pm E P^{b}$} & \multicolumn{7}{|c|}{ Percentil } \\
\hline & & & 10 & 15 & 25 & 50 & 75 & 85 & 90 \\
\hline \multicolumn{10}{|l|}{ CB (cm) } \\
\hline $60-69$ & 1122 & $31,7 \pm 0,21$ & 26,2 & 26,9 & 28,3 & 31,2 & 34,3 & 36,5 & 38,3 \\
\hline $70-79$ & 914 & $30,5 \pm 0,23$ & 25,4 & 26,1 & 27,4 & 30,1 & 33,1 & 35,1 & 36,7 \\
\hline$\geq 80$ & 712 & $28,5 \pm 0,25$ & 23,0 & 23,8 & 25,5 & 28,4 & 31,5 & 33,2 & 34,0 \\
\hline \multicolumn{10}{|l|}{ PCT (mm) } \\
\hline $60-69$ & 1090 & $24,2 \pm 0,37$ & 14,5 & 15,9 & 18,2 & 24,1 & 29,7 & 32,9 & 34,9 \\
\hline $70-79$ & 902 & $22,3 \pm 0,39$ & 12,5 & 14,0 & 16,4 & 21,8 & 27,7 & 30,6 & 32,1 \\
\hline$\geq 80$ & 705 & $18,6 \pm 0,42$ & 9,3 & 11,1 & 13,1 & 18,1 & 23,3 & 26,4 & 28,9 \\
\hline \multicolumn{10}{|l|}{$\mathrm{CMB}(\mathrm{cm})$} \\
\hline $60-69$ & 190 & $23,8 \pm 0,12$ & 20,6 & 21,1 & 21,9 & 23,5 & 25,4 & 26,6 & 27,4 \\
\hline $70-79$ & 898 & $23,4 \pm 0,14$ & 20,3 & 20,8 & 21,6 & 23,0 & 24,8 & 26,3 & 27,0 \\
\hline$\geq 80$ & 703 & $22,7 \pm 0,16$ & 19,3 & 20,0 & 20,9 & 22,6 & 24,5 & 25,4 & 26,0 \\
\hline
\end{tabular}

(a) Todos os grupos étnicos; ${ }^{(b)}$ Erro-padrão. $C B=$ circunferência do braço; $\mathrm{PCT}=$ prega cutânea tricipital; $\mathrm{CMB}=$ circunferência muscular do braço. Fonte: Kuczmarski et al. ${ }^{15}$. 
Quadro 1.Principais fatores que interferem no estado nutricional do idoso.

Relacionados à idade: $\begin{aligned} & \text { Sensibilidade olfativa e gustativa } \\ & \text { diminuída } \\ & \text { Supressão do apetite }\end{aligned}$

Dietéticos: Ingestão inadequada de alimentos

Médicos: Doenças

Efeitos farmacológicos

Problemas na cavidade oral

Incapacidade funcional

Psiquiátricos: Demência

Depressão

Distúrbio de comportamento

Anorexia nervosa

Alcoolismo

Sociais: Pobreza

Isolamento emocional

Viver só

Conhecimento nutricional insuficiente

Estilo de vida: Redução da atividade física

Tabagismo

Fonte: Modificado de Morigutti ${ }^{30}$.

Conclui-se, portanto, que a avaliação nutricional do idoso necessita ser realizada a partir de uma associação de indicadores, tendo em vista que cada um apresenta limitações, as quais devem ser complementadas. Os critérios de diagnóstico e dados de referência devem ser bastante específicos em razão das alterações que geralmente acompanham o processo de envelhecimento.

\section{REFER Ê N CIAS}

1. Ramos LR, Veras RP, Kalache A. Envelhecimento populacional: uma realidade brasileira. Rev Saude Publica 1987; 21:211-24.

2. Gariballa SE, Sinclair AJ. Nutrition, aging and ill health. Br J Nutr 1998; 80:7-23.

3. Vellas BJ, Alberede JL, Garry PJ. Diseases and aging: Patterns of morbidity with age; relationship between aging and age-associated diseases. Am J Clin Nutr 1992; 55:1225S-30S.
4. Najas MS, Sachs A. Avaliação nutricional do idoso. In: Papaléo Netto, M. Gerontologia. São Paulo: Atheneu; 1996. p.242-7.

5. Perissinotto E, Pisent C, Sergi G, Grigoletto F, Enzi G. Anthropometric measurements in the elderly: Age and gender differences. Br J Nutr 2002; 87:177-86.

6. Sullivan DH, Sun S, Walls RC. Protein-energy undernutrition among elderly hospitalized patients: A prospective study. JAMA 1999; 281:2013-9.

7. Kamimura MA, Baxmann A, Sampaio LR, Cuppari L. Avaliação nutricional. In: Cuppari L. Nutrição: nutrição clínica no adulto. São Paulo: Manole; 2002. p.71-109.

8. Coelho AC, Fausto MA. Avaliação pelo nutricionista. In: Maciel A. Avaliação multidisciplinar do paciente geriátrico. Rio de Janeiro: Revinter; 2002. p.121-56.

9. Goodman-Gruen D, Barret-Connor E. Sex differences in measures of body fat and body fat distribution in the elderly. Am J Epidemiol 1996; 143(9):898-906.

10. Chumlea WC, Roche AF, Steinbaugh ML. Estimating stature from knee height for persons 60 to 90 years of age. J Am Geriatr Soc 1985; 33:116-20.

11. Chumlea WC, Guo S, Roche AF, Steinbaugh ML. Prediction of body weight for the nonambulatory elderly from anthropometry. J Am Diet Assoc 1988; 88:564-8.

12. Going S, Williams D, Lohman T. Aging and body composition: Biological changes and methodological issues. Exerc Sport Sci Rev 1995; 23:411-58.

13. Dey DK, Rothenberg E, Sundh V, Bosaeus I, Steen B. Height and body weight in the elderly. I. A 25 year longitudinal study of a population aged 70 to 95 years. Eur J Clin Nutr 1999; 53:905-14.

14. Steen B. Body composition and aging. Nutr Rev 1988; 46:45-51.

15. Kuczmarski MF, Kuczarisk RJ, Najjar M. Descriptive anthropometric reference data for older Americans. J Am Diet Assoc 2000; 100:59-66. 
16. World Health Organization. Physical Status: The use and interpretation of anthropometry. Geneva; 1995. (WHO Technical Report Series, 854).

17. Frisancho AR. New norms of upper limb fat and muscle areas for assessment of nutritional status. Am J Clin Nutr 1981; 34:2540-45.

18. Enzi G, Gasparo M, Biondetti PR, Fiore D, Semisa M, Zurlo F. Subcutaneous and visceral fat distribution according to sex, age and overweight, evaluated by computed tomography. Am J Clin Nutr 1986; 44:739-46.

19. Pouliot M-C, Després J-P, Lemieux S, Moorjani S, Bouchard C, Tremblay A, et al. Waist circunference and abdominal sagittal diameter: Best simple anthropometric indexes of abdominal visceral adipose tissue accumulation and related cardiovascular risk in men and women. Am J Cardiol $1994 ; 73(1): 460-8$.

20. Han TS, Seidell JC, Curral JEP, Morrison CE, Deurenberg $P$, Lean MEJ. The influences of height and age on waist circunference as an index of adiposity in adults. Int J Obes 1997; 21:83-9.

21. DiPietro L, Katz LD, Nadel ER. Excess abdominal adiposity remains correlated with altered lipid concentrations in healthy older women. Int J Obes Related Metabol Disord 1999; 432-6.

22. Han TS, van Leer EM, Seidell JC, Lean MEJ. Waist circunference action levels in the identification of cardiovascular risk factors: Prevalence study in a random sample. BMJ 1995; 311:1401-5.

23. Seidell JC, Kahn HS, Williamson DF, Lisser L, Valdez R. Report from a Centers for Disease Control and
Prevention workshop on use of adult anthropometry for public health and primary health care. Am J Clin Nutr 2001; 73:123-6.

24. World Health Organization. Obesity: Preventing and managing the global epidemic. Geneva; 1997.

25. Seidell JC, Oosterlee A, Deurenberg P, Hautvast JAGJ, Ruijs JHJ. Abdominal fat depots measured with computed tomography: effects of degree of obesity, sex, and age. Eur J Clin Nutr 1988; 42:805-15.

26. Bray GA. Classification and evaluation of the obesities. Med Clin North Am 1989; 73:161-84.

27. Kahn HS, Austin H, Williamson DF, Arensberg D. Simple anthropometric indices associated with ischemic heart disease. J Clin Epidemiol 1996; 49(9):1017-24.

28. Frisancho AR. New standards of weight and body composition by frame size and height for assessment of nutritional status of adults and the elderly. Am J Clin Nutr 1984; 40:808-19.

29. Chumlea WC, Roche AF, Mukherjee D. Nutritional assessment of the elderly through anthropometry. Columbus, Ohio: Ross Laboratories; 1987.

30. Morigutti JC, Morigutti EKU, Ferrioli E, et al. Involuntary weight loss in elderly individuals: Assessment and treatment. Med J 2001; 119(2): 72-7.

Recebido para publicação em 30 de janeiro e aceito em 13 de novembro de 2003. 